\title{
Review \\ Organic chemicals in sewage sludges
}

\author{
Ellen Z. Harrison ${ }^{\mathrm{a}, *}$, Summer Rayne Oakes ${ }^{\mathrm{a}}$, Matthew Hysell ${ }^{\mathrm{a}}$, Anthony Hay ${ }^{\mathrm{b}}$ \\ a Cornell Waste Management Institute, Department of Crop and Soil Sciences, Rice Hall, Ithaca, NY 14853, United States \\ ${ }^{\mathrm{b}}$ Cornell University, Department of Microbiology and Institute for Comparative and Environmental Toxicology, Ithaca, NY 14853, United States
}

Received 6 June 2005; received in revised form 4 April 2006; accepted 18 April 2006

Available online 5 June 2006

\begin{abstract}
Sewage sludges are residues resulting from the treatment of wastewater released from various sources including homes, industries, medical facilities, street runoff and businesses. Sewage sludges contain nutrients and organic matter that can provide soil benefits and are widely used as soil amendments. They also, however, contain contaminants including metals, pathogens, and organic pollutants. Although current regulations require pathogen reduction and periodic monitoring for some metals prior to land application, there is no requirement to test sewage sludges for the presence of organic chemicals in the U. S. To help fill the gaps in knowledge regarding the presence and concentration of organic chemicals in sewage sludges, the peer-reviewed literature and official governmental reports were examined. Data were found for 516 organic compounds which were grouped into 15 classes. Concentrations were compared to EPA risk-based soil screening limits (SSLs) where available. For 6 of the 15 classes of chemicals identified, there were no SSLs. For the 79 reported chemicals which had SSLs, the maximum reported concentration of $86 \%$ exceeded at least one SSL. Eighty-three percent of the 516 chemicals were not on the EPA established list of priority pollutants and $80 \%$ were not on the EPA's list of target compounds. Thus analyses targeting these lists will detect only a small fraction of the organic chemicals in sludges. Analysis of the reported data shows that more data has been collected for certain chemical classes such as pesticides, PAHs and PCBs than for others that may pose greater risk such as nitrosamines. The concentration in soil resulting from land application of sludge will be a function of initial concentration in the sludge and soil, the rate of application, management practices and losses. Even for chemicals that degrade readily, if present in high concentrations and applied repeatedly, the soil concentrations may be significantly elevated. The results of this work reinforce the need for a survey of organic chemical contaminants in sewage sludges and for further assessment of the risks they pose.
\end{abstract}

(C) 2006 Elsevier B.V. All rights reserved.

Keywords: Sludge; Biosolids; Land application

\section{Contents}

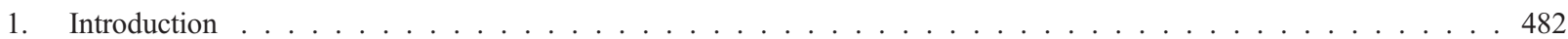

2. Methods . . . . . . . . . . . . . . . . . . . . . . . . . . . . . . 483

3. Results and discussion . . . . . . . . . . . . . . . . . . . . . . . . . . . . 491

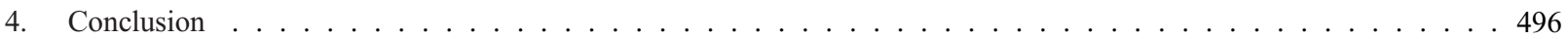

Appendix A. Supplementary data . . . . . . . . . . . . . . . . . . . . . . . . . . . 496

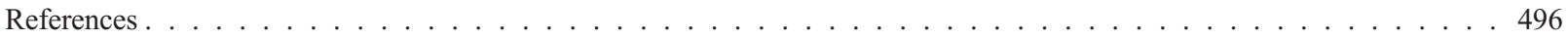

* Corresponding author. Tel.: +1 607255 8576; fax: +1 6072558207.

E-mail address: ezh1@cornell.edu (E.Z. Harrison). 


\section{Introduction}

Sewage sludges are residues generated at centralized wastewater treatment plants (WWTPs) as a result of the treatment of wastes released from a variety of sources including homes, industries, medical facilities, street runoff and businesses The use of these sludges as soil amendments is widely practiced in the U.S., where more than $60 \%$ of the 6.2 million dry metric tons (MT) of sludge produced annually are applied to land (U.S. Environmental Protection Agency, 1999). Since 1991 when ocean dumping was banned, both the quantity produced and the percentage land-applied have increased (U.S. Environmental Protection Agency, 1999).

Sewage sludges contain nutrients and organic matter that can provide soil benefits, but they also contain contaminants including metals, pathogens, and organic pollutants. The fate of chemical contaminants entering a WWTP depends on both the nature of the chemical and the treatment processes (Zitomer and Speece, 1993). Organic chemicals may be volatilized, degraded (through biotic and/or abiotic processes), sorbed to sludge, or discharged in the aqueous effluent. Degradation results in the creation of breakdown products that can be either more or less toxic than the original compound.

For many hydrophobic organic chemicals, sorption to the sewage sludge solids is the primary pathway for their removal from wastewater. This is especially true of persistent, bioaccumulative toxics that may enter the waste stream (Petrasek et al., 1983). Even volatile chemicals, such as benzene, are commonly found in sewage sludges as a result of sorption to organic substances in the sludge matrix (Wild et al., 1992). After they have been separated from wastewater, landapplied sludges must be treated to reduce pathogens through one of a number of processes including anaerobic digestion, lime stabilization, or composting. Each of these processes has effects on the fate of both pathogens and the organic contaminants in the sludge (Rogers, 1996).

The information available on the concentration of organic chemicals in sewage sludges arises largely from academic reports or from the national sewage sludge survey (NSSS) which was conducted by the U.S. Environmental Protection Agency (EPA) in 1988 (U.S. Environmental Protection Agency, 1990). The NSSS was performed by analyzing samples of the final sludge product collected from approximately 180 wastewater plants for the presence of 411 chemicals. This survey was used in the development of the U.S. regulations (U.S. Environmental Protection Agency, 1996).
Very few countries have rules limiting the concentration of any organic chemicals in sewage sludges (Beck et al., 1995). The European Union is considering establishing limits for a handful of organic chemicals. Under the Clean Water Act, (CFR Section 405 (d)), the rules regarding the concentration of pollutants permitted in land-applied sewage sludges in the U.S. are mandated to be protective of human health and the environment. A biennial review is called for to determine if there are additional chemicals that might pose a risk and should thus be subject to regulatory review.

To date, EPA has not established regulations for any organic chemicals and there is no federal requirement to monitor the type or concentration of organic chemicals in sludges. When promulgating the original rules in 1993 (CFR 40 Part 503), the EPA declined to include any organic contaminants. There were three criteria that led to the elimination of all of those considered: 1 . the chemical was no longer in use in the U.S.; 2. the chemical was detected in $5 \%$ or fewer of the sludges tested in the NSSS; or 3. a hazard screening showed the chemical to have a hazard index of one or greater (Beck et al., 1995). Where sufficient data were lacking to evaluate the hazard, for example the lack of fate and transport data, that chemical and pathway were also eliminated from further consideration (U.S. Environmental Protection Agency, 1996).

Concerns with this process include the persistence of some chemicals in the environment despite their elimination in commerce, the high detection limits for some chemicals, and the potential risks posed by chemicals that were eliminated from consideration merely due to a lack of data (National Research Council, 2002). In a court-ordered review of additional contaminants, the EPA reconsidered regulation of some organic chemicals. In that review, it eliminated chemicals that were detected in $10 \%$ or fewer of the sludges in the NSSS. Of the 411 analytes in the NSSS 269 were not detected and 69 were detected in fewer than $10 \%$ of the sludges. Fifteen of the 73 remaining chemicals were eliminated due to lack of toxicity data (U.S. Environmental Protection Agency, 1996). Hazard screening analysis was conducted on the remaining chemicals. Dioxins, furans and co-planar PCBs were the only organic chemicals that remained and a risk assessment was then conducted (U.S. Environmental Protection Agency, 2002). Based on the assessment, EPA decided not to extend regulation to dioxins or any other organic pollutant (U.S. Environmental Protection Agency, 2003a). The Round 2 review conducted by the EPA in 2003 was not limited to the chemicals analyzed in the 
NSSS. It considered 803 chemicals and resulted in the selection of 15 chemicals as candidates for regulation based on available human health or ecological risk end points but not on concentration data from sludges. Among those were 9 organic chemicals (U.S. Environmental Protection Agency, 2003b).

The National Research Council of the U.S. Academy of Sciences (NRC) conducted two reviews of the land application of biosolids (National Research Council, 1996; 2002). Their 2002 report included a comparison of the limits of detection for samples analyzed in the NSSS to EPA soil screening limits (SSLs) and pointed out that high limits of detection for many chemicals in the NSSS were a concern. The SSLs are conservative risk-based soil concentrations of selected industrial pollutants (93 organic and 16 inorganic compounds) that are used in determining whether a site specific risk assessment is required at a Superfund site (U.S. Environmental Protection Agency Superfund, 1996).

The SSLs were used by the NRC as an indicator of concentrations that might pose a risk requiring remediation. For 5 of 8 organic chemicals examined in the NRC report, most sludge samples analyzed in the NSSS had limits of detection that were higher than the EPAestablished SSLs. Thus the NSSS results were not sensitive enough to detect pollutant concentrations that, if present in soil at a Superfund site, would have triggered a risk assessment. For example, in the case of hexachlorobenzene (HCB), the NSSS did not detect HCB in any of the 176 samples tested, thus prompting EPA to exclude it from regulatory consideration. The NSSS limits of detection exceeded $5 \mathrm{mg} / \mathrm{kg}$ for the majority of samples and was greater than $100 \mathrm{mg} / \mathrm{kg}$ for 4 samples (National Research Council, 2002). Depending on the pathway of exposure being considered, the SSLs for HCB range from 0.1 to $2 \mathrm{mg} / \mathrm{kg}$. Only one of the NSSS samples reached a limit of detection of $0.1 \mathrm{mg} / \mathrm{kg}$. Analysis of the data compiled in this paper revealed that 9 of the 13 reports of $\mathrm{HCB}$ concentrations in sewage sludges exceeded $0.1 \mathrm{mg} / \mathrm{kg}$ and 3 exceeded $2 \mathrm{mg} / \mathrm{kg}$. Thus the majority of samples exceeded an SSL for HCB.

In addition to concerns regarding analytical limitations, the introduction of new chemicals into commerce, suggests that there is a need for a new survey in order to better characterize sludges with respect to the presence and concentration of contemporary organic chemicals. Flame retardants, surfactants, chlorinated paraffins, nitro and polycyclic musks, pharmaceuticals, odorants, as well as chemicals used in treating sludges (such as dewatering agents) are among the chemical categories suggested by the NRC as compounds requiring additional data collection and consideration in future risk assessments (National Research Council, 2002).

Although the EPA conducted a limited survey of sludges in 2001 to determine the concentration of dioxins, furans and co-planar PCBs, and plans to conduct a survey of sludges to test for the 9 organic chemicals being considered for regulation, it is not proposing a broader survey of organic chemicals in sludges (U.S. Environmental Protection Agency, 2003b).

\section{Methods}

To help fill the gaps in knowledge regarding the presence and concentration of organic chemicals in sewage sludges, we examined the peer-reviewed literature and official governmental reports to compile available data on the concentration of organic chemicals reported in sludges. In some cases sources did not contain sufficient information to permit comparison of chemical concentrations as a function of sludge dry weight and were therefore not included. One hundred and thirteen usable data sets were obtained. Reports were inconsistent in providing individual versus average or median values so we have reported the ranges detected and are not able to offer averages. Where available, average values from a specific report are noted (supporting information 1). There are several important aspects of wastewater and sludge treatment that can affect the fate of organic chemicals. Unfortunately many reports do not include such information. Where available, the type of treatment is noted (supporting information 1). Similarly, most reports did not include information on the type of catchment area or on significant non-domestic inputs that might contribute particular chemicals.

The chemicals were grouped into 15 classes and the range of concentrations reported for each chemical was recorded. Data were found for 516 chemicals and the range of concentrations detected in each of the sources was recorded (supporting information 1). For ease of presentation, this list was reduced to 267 chemicals through the grouping of congeners and isomeric compounds. The range of concentrations for compounds that have been reported in sewage sludges and the sources from which these data were obtained are shown in Table 1.

To provide a context for the sludge concentration data, we sought soil pollutant concentration standards with which to compare the sludge concentrations. We found that the U.S. SSLs, soil clean-up standards in Ontario and Dutch Intervention values were supported 
Table 1

Concentrations of organic chemicals reported in sewage sludges and sources of those data

\begin{tabular}{|c|c|c|}
\hline & \multirow{2}{*}{$\frac{\text { Range }}{\mathrm{mg} / \mathrm{kg} \text { dry wgt }}$} & \multirow{2}{*}{$\begin{array}{l}\text { Data } \\
\text { sources }^{a}\end{array}$} \\
\hline & & \\
\hline \multicolumn{3}{|c|}{ Aliphatics-short chained and chlorinated } \\
\hline Acrylonitrile & $0.0363-82.3$ & [1] \\
\hline $\begin{array}{l}\text { Butadiene } \\
\quad(\text { hexachloro-1,3-) }\end{array}$ & ND-8 & {$[1-4]$} \\
\hline Butane (1,2,3,4-diepoxy) & ND-73.9 & {$[5]$} \\
\hline Butanol (iso) & ND-0.165 & {$[5]$} \\
\hline Butanone (2-) & ND-1540 & {$[5]$} \\
\hline Carbon disulfide $\mathrm{SSL}^{\mathrm{SS}}$ & ND-23.5 & {$[5]$} \\
\hline Crotonaldehyde & ND-0.358 & {$[5]$} \\
\hline $\begin{array}{l}\text { Cyclopentadiene } \\
\text { (hexachloro) }^{\mathrm{SSL}}\end{array}$ & $<0.005$ & {$[2]$} \\
\hline Ethane (hexachloro) ${ }^{\mathrm{SSL}}$ & $0.00036-61.5$ & {$[3]$} \\
\hline Ethane (monochloro) & ND-24 & [3] \\
\hline Ethane (pentachloro) & $0.0003-9.2 \mathrm{~g}$ & {$[3]$} \\
\hline Ethane (tetrachloro) & $<0.1-5.0$ & {$[6]$} \\
\hline $\begin{array}{l}\text { Ethane (trichloro) } \\
\text { isomers }\end{array}$ & ND-33 & {$[7]$} \\
\hline Ethylene (dichloro) ${ }^{\text {SSL }}$ & $<0.01-865$ & {$[3,8]$} \\
\hline Ethylene (monochloro) & $<0.025-110$ & {$[2,3]$} \\
\hline Ethylene (tetrachloro) ${ }^{\text {SSL }}$ & ND-50 & {$[1-3,5,7,8]$} \\
\hline Ethylene (trichloro) ${ }^{\text {SŚL }}$ & ND-125 & {$[2,3,5,7]$} \\
\hline Hexanoic acid & ND-1960 & {$[5]$} \\
\hline Hexanone (2-) & ND-12.7 & {$[5]$} \\
\hline Methane (dichloro) ${ }^{\mathrm{SSL}}$ & ND-262 & {$[3,5,8,9]$} \\
\hline Methane (monochloro) & ND-30 & [5] \\
\hline Methane (tetrachloro) $^{\mathrm{SSL}}$ & ND-60 & {$[2,3,5-7]$} \\
\hline Methane (trichloro) ${ }^{\mathrm{SŚ} L}$ & ND-60 & {$[2,5-7]$} \\
\hline Methane (trichlorofluoro) & ND-3.97 & {$[5]$} \\
\hline$N$-alkanes (polychlorinated) & $1.8-93.1$ & {$[10]$} \\
\hline$N$-alkanes & ND-758 & {$[5]$} \\
\hline $\begin{array}{l}\text { Organic halides absorbable } \\
(\text { AOX) and extractable } \\
\text { (EOX) }\end{array}$ & $1-7600$ & {$[7,11-13]$} \\
\hline Pentanone (methyl) & ND- 0.567 & {$[5]$} \\
\hline Polyorganosiloxanes & $8.31-5155$ & {$[14-18]$} \\
\hline $\begin{array}{l}\text { Propane (dichloro) } \\
\text { isomers } \mathrm{SSL}\end{array}$ & ND-1230 & {$[1,3,5]$} \\
\hline Propane (trichloro) & $0.00459-19.5$ & {$[1,3]$} \\
\hline $\begin{array}{l}\text { Propanenitrile } \\
\text { (ethyl cyanide) }\end{array}$ & ND-64.7 & {$[5]$} \\
\hline Propanone (2-) & ND-2430 & {$[5]$} \\
\hline Propen-1-ol (2-) & ND-0.0312 & {$[5]$} \\
\hline Propene (trichloro) & $<0.0010-167$ & {$[1]$} \\
\hline $\begin{array}{l}\text { Propene chlorinated } \\
\text { isomers }\end{array}$ & $0.002-1230$ & {$[3,5]$} \\
\hline Propenenitrile (methyl) & ND-218 & {$[5]$} \\
\hline Squalene & ND-16.7 & {$[5]$} \\
\hline Sulfone (dimethyl) & ND-0.784 & {$[5]$} \\
\hline \multicolumn{3}{|l|}{ Chlorobenzenes } \\
\hline $\begin{array}{l}\text { Benzene (dichloro) } \\
\text { isomers }\end{array}$ & ND-1650 & $\begin{array}{l}{[2,3,5,8,} \\
19,20]\end{array}$ \\
\hline Benzene (hexachloro) ${ }^{\mathrm{SSL}}$ & ND-65 & $\begin{array}{l}{[1,2,4,7,11,} \\
20-22]\end{array}$ \\
\hline Benzene (monochloro) ${ }^{\mathrm{SSL}}$ & ND-846 & {$[3,5,19]$} \\
\hline
\end{tabular}

Table 1 (continued)

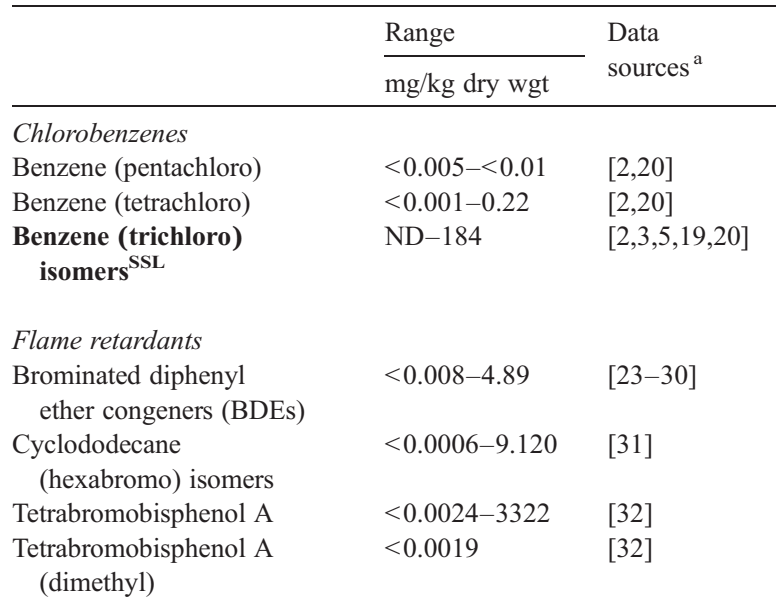

Monocyclic hydrocarbons and heterocycles

Acetophenone ND-6.92

Aniline (2,4,5-trimethyl) ND-0.220

Benzene $^{\text {SSL }}$

ND-11.3

Benzene (1,4-dinitro)

Benzene (ethyl) ${ }^{\text {SSL }}$

Benzene (mononitro) $)^{\text {SSL }}$

ND-4.4

ND-65.5

Benzene (trinitro)

ND-1.55

12

ND-64.4

Benzenethiazole

(2-methylthio)

Benzenethiol

ND-3.25

Benzoic acid ${ }^{\text {SSL }}$

Benzyl alcohol

Analine (chloro) $(\mathrm{P}-)^{\mathrm{SSL}}$

Cymene (P-)

Dioxane (1,4-)

Picoline (2-)

Styrene $^{\text {SSL }}$

Terpeniol (alpha)

Thioxanthe-9-one

Toluene ${ }^{\text {SSL }}$

ND-835

ND-156

ND-40.2

[5]

[5]

$[3,5,33]$

[5]

$[3,5]$

$[2,5]$

[34]

[5]

[5]

[5]

[5]

[5]

ND-84.3

ND-35.3

ND-365

ND-5850

ND-2.56

ND-19.6

ND-1180

Toluene (chloro)

1.13-324

Toluene (2,4-dinitro) $)^{\text {SSL }}$

Toluene (para nitro)

ND-10

Toluene (trinitro)

Xylene isomers ${ }^{\mathrm{SSL}}$

100

12

ND-6.91

[5]

[5]

[5]

$[3,5]$

[5]

[5]

$[3,5,6,8,9$, $34,35]$

[5]

$[2,5,34]$

[34]

[34]

$[5,8,33$,

35-37]

Nitrosamines

$\mathrm{N}$-nitrosdiphenylamine $\mathrm{SSL}^{\mathrm{SL}}$

ND-19.7

[5]

$\mathrm{N}$-nitrosodiethylamine

ND-0.0038

[38]

$\mathrm{N}$-nitrosodimethylamine

$N$-nitrosodi- $n$-butylamine

$\mathrm{N}$-nitrosomorpholine

0.0006-0.053

[38]

ND

ND-0.0092 [38]

$\mathrm{N}$-nitrosopiperdine

$\mathrm{N}$-nitrosopyrrolidine

ND-trace

ND- 0.0042

[38]

Organotins

Butylitin (di)

$0.41-8.557$

[39-44]

Butyltin (mono) 
Table 1 (continued)

\begin{tabular}{|c|c|c|}
\hline & \multirow{2}{*}{$\frac{\text { Range }}{\mathrm{mg} / \mathrm{kg} \text { dry wgt }}$} & \multirow{2}{*}{$\begin{array}{l}\text { Data } \\
\text { sources }^{\text {a }}\end{array}$} \\
\hline & & \\
\hline \multicolumn{3}{|l|}{ Organotins } \\
\hline Butyltin (tri) & $0.005-237.923$ & {$[9,39-44]$} \\
\hline Phenyltin (di) & $0.1-0.4$ & {$[42,43]$} \\
\hline Phenyltin (mono) & 0.1 & {$[42,43]$} \\
\hline Phenyltin (tri) & $0.3-3.4$ & {$[42,43]$} \\
\hline \multicolumn{3}{|c|}{ Personal care products and pharmaceuticals } \\
\hline Acetaminophen & $0.0000006-4.535$ & {$[45]$} \\
\hline Gemfibrozil & ND-1.192 & {$[45]$} \\
\hline Ibuprofen & $0.000006-3.988$ & {$[45]$} \\
\hline Naproxen & $0.000001-1.022$ & {$[45]$} \\
\hline Salicylic acid & $0.000002-13.743$ & {$[45]$} \\
\hline \multicolumn{3}{|l|}{ Antibiotics } \\
\hline Ciprofloxacin & $0.05-4.8$ & {$[46,47]$} \\
\hline Doxycycline & $<1.2-1.5$ & {$[47]$} \\
\hline Norfloxacin & $0.01-4.2$ & {$[46,47]$} \\
\hline Ofloxacin & $<0.01-2$ & {$[47]$} \\
\hline $\begin{array}{l}\text { Triclosan (4-chloro- } \\
\text { 2-(2,4-dichloro- } \\
\text { phenoxy)-phenol and } \\
\text { related compounds }\end{array}$ & ND-15.6 & {$[25,48-50]$} \\
\hline \multicolumn{3}{|l|}{ Fluorescent whitening agents } \\
\hline $\begin{array}{l}\text { BLS (4,4'-bis(4- } \\
\text { chloro-3-sulfostyryl)- } \\
\text { biphenyl) }\end{array}$ & $5.4-5.5$ & {$[51]$} \\
\hline $\begin{array}{l}\text { DAS } 1 \text { (4,4'- } \\
\text { bis[(4-anilino-6- } \\
\text { morpholino-1,3,5- } \\
\text { triazin-2-yl)-amino] } \\
\text { stilbene-2,2'-disulfonate) }\end{array}$ & $86-112$ & {$[51]$} \\
\hline $\begin{array}{l}\text { DSBP (4,4'-bis } \\
\text { (2-sulfostyryl)biphenyl) }\end{array}$ & $31-50$ & {$[51]$} \\
\hline \multicolumn{3}{|l|}{ Fragrance material } \\
\hline Acetyl Cedrene & $9.0-31.1$ & {$[52]$} \\
\hline Amino Musk Ketone & ND-0.362 & {$[37]$} \\
\hline $\begin{array}{l}\text { Amino Musk Xylene } \\
(\text { AMX) }\end{array}$ & ND-0.0315 & {$[37]$} \\
\hline $\begin{array}{l}\text { Cashmeran (DPMI) } \\
\text { (6,7-dihydro-1,1,2,3,3- } \\
\text { pentamethyl-4(5H)- } \\
\text { indanone) }\end{array}$ & ND-0.332 & {$[34,37]$} \\
\hline $\begin{array}{l}\text { Celestolide (1-[6- } \\
\text { (1,1-Dimethylethyl)- } \\
\text { 2,3-dihydro-1,1-methyl- } \\
\text { 1H-inden-4-yl]-ethanone) }\end{array}$ & $0.010-1.1$ & {$[34,37,53,54]$} \\
\hline Diphenyl Ether & ND-99.6 & {$[5,52]$} \\
\hline $\begin{array}{l}\text { Galaxolide (HHCB) } \\
(1,3,4,6,7,8 \text {-Hexahydro- } \\
4,6,6,7,8,8- \\
\text { hexamethylcyclopenta[g]- } \\
\text { benzopyran) }\end{array}$ & ND-81 & $\begin{array}{l}{[25,34,37,} \\
52-56]\end{array}$ \\
\hline $\begin{array}{l}\text { Galaxolide lactone } \\
(1,3,4,6,7,8 \text {-Hexahydro- } \\
\text { 4,6,6,7,8,8- } \\
\text { hexamethylcyclopenta[g]- } \\
\text { 2-benzopyran-1-one) }\end{array}$ & $0.6-3.5$ & {$[54]$} \\
\hline Hexyl salicylate & Trace- -1.5 & {$[52]$} \\
\hline
\end{tabular}

Table 1 (continued)

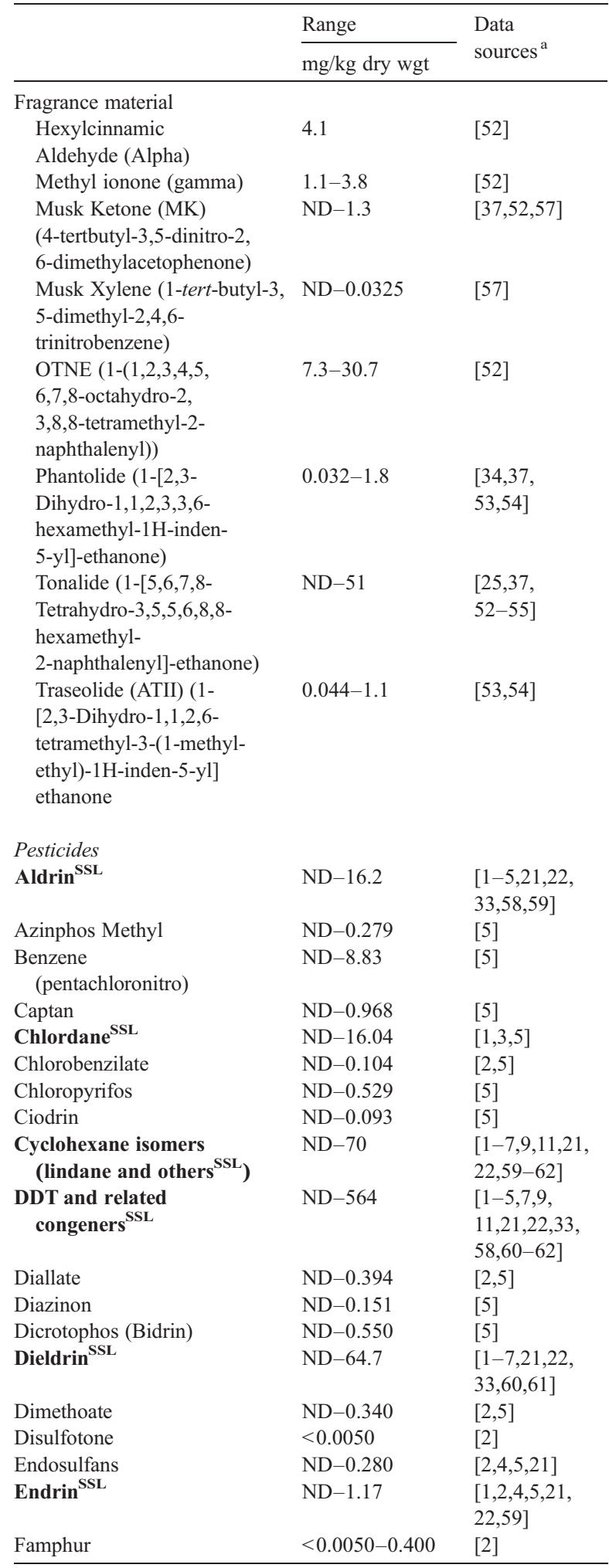


Table 1 (continued)

\begin{tabular}{|c|c|c|}
\hline & \multirow{2}{*}{$\frac{\text { Range }}{\mathrm{mg} / \mathrm{kg} \text { dry wgt }}$} & \multirow{2}{*}{$\begin{array}{l}\text { Data } \\
\text { sources }^{a}\end{array}$} \\
\hline & & \\
\hline \multicolumn{3}{|l|}{ Pesticides } \\
\hline Heptachlor epoxides ${ }^{\text {SSL }}$ & $\mathrm{ND}-0.780$ & {$[1,2,5,21]$} \\
\hline Heptachlor $^{\text {SSL }}$ & ND-16 & {$[2,3,5,21,22]$} \\
\hline Isobenzan & $\mathrm{ND}-0.130$ & {$[4]$} \\
\hline Isodrin & ND & {$[4]$} \\
\hline Isophorone $^{\text {SSL }}$ & $<0.0050-0.08294$ & {$[2]$} \\
\hline Leptophos & ND-0.319 & {$[5]$} \\
\hline Methoxychlor ${ }^{\text {SSL }}$ & $<0.015-0.330$ & {$[2]$} \\
\hline Mevinphos (phosdrin) & ND-0.148 & {$[5]$} \\
\hline Naled (Dibrom) & ND-0.484 & {$[5]$} \\
\hline Naphthoquinone $(1,4-)$ & $<0.0050$ & {$[2]$} \\
\hline Nitrofen & $\mathrm{ND}-0.195$ & {$[5]$} \\
\hline Parathion (ethyl) & $<0.0050-0.380$ & {$[2]$} \\
\hline Parathion (methyl) & $<0.0050-0.070$ & {$[2]$} \\
\hline Permethrin isomers & $<0.15-163$ & {$[20,63]$} \\
\hline Phenoxy herbicides ${ }^{\text {SSL }}$ & ND-7.34 & {$[1,2,5]$} \\
\hline $\begin{array}{l}\text { Phenoxypropanoic } \\
\text { acid (trichloro) }\end{array}$ & $\mathrm{ND}-0.121$ & {$[5]$} \\
\hline $\begin{array}{l}\text { Phorate }(O, O \text {-diethyl } \\
S \text {-[(ethylthio) } \\
\text { methyl] } \\
\text { phosphorodithioate })\end{array}$ & $<0.0050-0.200$ & {$[2]$} \\
\hline Phosphamidon & $\mathrm{ND}-0.232$ & {$[5]$} \\
\hline $\begin{array}{l}\text { Pronamide (dichloro } \\
\text { (3,5-)- } N \text {-(1,1- } \\
\text { dimethylpropynyl) } \\
\text { benzamide) }\end{array}$ & $<0.0050-0.008$ & {$[2]$} \\
\hline $\begin{array}{l}\text { Pyrophosphate } \\
\text { (tetraethyl) }\end{array}$ & ND-20 & {$[5]$} \\
\hline Quintozene & $\mathrm{ND}-0.100$ & {$[4]$} \\
\hline Safrol (iso) & $<0.0050-0.750$ & {$[2]$} \\
\hline Safrole (EPN) & ND-0.545 & {$[2]$} \\
\hline Toxaphene $\mathrm{SSL}^{\mathrm{SS}}$ & 51 & [3] \\
\hline Trichlorofon & ND-2.53 & {$[5]$} \\
\hline Trifluralin (Treflan) & ND- -0.235 & {$[5]$} \\
\hline \multicolumn{3}{|l|}{ Phenols } \\
\hline Bisphenol-A (BPA) & $0.00010-32,100$ & {$[18,49,64,65]$} \\
\hline Hexachlorophene (HCP) & $0.0226-1.190$ & [49] \\
\hline Hydroquinone & $0.14-223$ & {$[3]$} \\
\hline Hydroxybiphenyls & $\mathrm{ND}-0.172$ & [64] \\
\hline Phenol $^{\text {SSL }}$ & ND-920 & $\begin{array}{l}{[2,3,5,7} \\
8,36,66]\end{array}$ \\
\hline $\begin{array}{l}\text { Phenol chloro } \\
\text { congeners }^{\text {SSL }}\end{array}$ & $<0.003-8490$ & $\begin{array}{l}{[1-3,5-9,} \\
33,35,49 \\
61,66-68]\end{array}$ \\
\hline $\begin{array}{l}\text { Phenol chloro methyl } \\
\text { congeners }\end{array}$ & ND-136 & $\begin{array}{l}{[2,3,5,8,9,} \\
61,64]\end{array}$ \\
\hline $\begin{array}{l}\text { Phenol methyl } \\
\text { congeners }\end{array}$ & ND-1160 & $\begin{array}{l}{[2,3,5,7-9} \\
34,66]\end{array}$ \\
\hline $\begin{array}{l}\text { Phenol nitro methyl } \\
\text { congeners }\end{array}$ & $0.2-187$ & {$[5]$} \\
\hline $\begin{array}{l}\text { Phenols nitro } \\
\text { congeners }\end{array}$ & $<0.003-500$ & {$[2,3,8]$} \\
\hline
\end{tabular}

Table 1 (continued)

\begin{tabular}{|c|c|c|}
\hline & Range & \multirow{2}{*}{$\begin{array}{l}\text { Data } \\
\text { sources }^{\text {a }}\end{array}$} \\
\hline & $\mathrm{mg} / \mathrm{kg}$ dry wgt & \\
\hline \multicolumn{3}{|c|}{ Phthalate acid esters/plasticizers } \\
\hline $\begin{array}{l}\text { Bis(2-chloroethyl) } \\
\text { ether }\end{array}$ & $<0.020-0.130$ & {$[2]$} \\
\hline $\begin{array}{l}\text { Bis(2-chloroisopropyl) } \\
\text { ether }\end{array}$ & $<0.150-5.700$ & [2] \\
\hline $\begin{array}{l}\text { Bis(2-cloroethoxy) } \\
\text { methane }\end{array}$ & $<0.020-0.240$ & {$[2]$} \\
\hline $\begin{array}{l}\text { Di(2-ethylhexyl) } \\
\text { adipate }\end{array}$ & $<0.100-0.450$ & [2] \\
\hline Phthalates ${ }^{\text {SSL }}$ & ND-58,300 & $\begin{array}{l}{[2,3,5-9,} \\
28,33,36 \\
58,69-73]\end{array}$ \\
\hline \multicolumn{3}{|c|}{ Polychlorinated biphenyls, naphthalenes, dioxins and furans } \\
\hline Aroclor 1016 & $0.2-75$ & {$[6,74]$} \\
\hline Aroclor 1248 & ND-5.2 & {$[5,6,33,58]$} \\
\hline Aroclor 1254 & $0.0667-1960$ & {$[1,5]$} \\
\hline Aroclor 1260 & ND-433 & {$[1,5,6,58,60]$} \\
\hline Biphenyl (decachloro) & $0.11-2.9$ & {$[1]$} \\
\hline $\begin{array}{l}\text { Biphenyls } \\
\text { (polybrominated) }\end{array}$ & 431 & {$[3]$} \\
\hline Dibenzofuran & ND-59.3 & {$[5]$} \\
\hline $\begin{array}{l}\text { Dioxins and furans } \\
\text { (polychlorinated } \\
\text { dibenzo) }\end{array}$ & ND-1.7 & $\begin{array}{l}{[5,8,72,} \\
75-81]\end{array}$ \\
\hline PCB congeners & ND-765 & $\begin{array}{l}{[2-5,7,11,} \\
13,21,22,28, \\
35,53,59, \\
61,71,72, \\
79,81-87]\end{array}$ \\
\hline Phenylether (chloro) & $<0.020$ & [2] \\
\hline $\begin{array}{l}\text { Terphenyls and } \\
\text { naphthalenes } \\
\text { (polychlorinated) }\end{array}$ & ND-11.1 & $\begin{array}{l}{[2,3,5,9,} \\
28,53]\end{array}$ \\
\hline \multicolumn{3}{|c|}{ Polynuclear aromatic hydrocarbons } \\
\hline Acenaphthene ${ }^{S S L}$ & ND-6.6 & $\begin{array}{l}{[2,5,8,21,53,} \\
82,88]\end{array}$ \\
\hline Acenaphthylene & $0.00360-0.3$ & {$[2,8,21,53]$} \\
\hline Anthracene $\mathrm{SSL}^{\mathrm{SS}}$ & ND-44 & $\begin{array}{l}{[2,3,5,8,21} \\
28,31,53 \\
74,88,89]\end{array}$ \\
\hline Benzidine & 12.7 & [3] \\
\hline $\operatorname{Benzo}(a)$ anthracene $^{\text {SSL }}$ & ND-99 & $\begin{array}{l}{[2,3,5,8,} \\
21,53, \\
82,88-90]\end{array}$ \\
\hline Benzo[ghi]perylene & ND-12.9 & $\begin{array}{l}{[1,2,5-8,} \\
21,22,28, \\
53,88-91]\end{array}$ \\
\hline $\begin{array}{l}\text { Benzofluoranthene } \\
\text { congeners }^{\text {SSL }}\end{array}$ & $0.006-34.2$ & {$[3,89]$} \\
\hline $\begin{array}{l}\text { Benzofluorene } \\
\text { congeners }\end{array}$ & ND-8.1 & {$[62,89]$} \\
\hline $\begin{array}{l}\text { Benzopyrene } \\
\text { congeners }^{\mathrm{SSL}}\end{array}$ & ND-24.7 & $\begin{array}{l}{[1-3,5-8,} \\
11,21,22,28, \\
33,53,62, \\
82,88-91]\end{array}$ \\
\hline
\end{tabular}


Table 1 (continued)

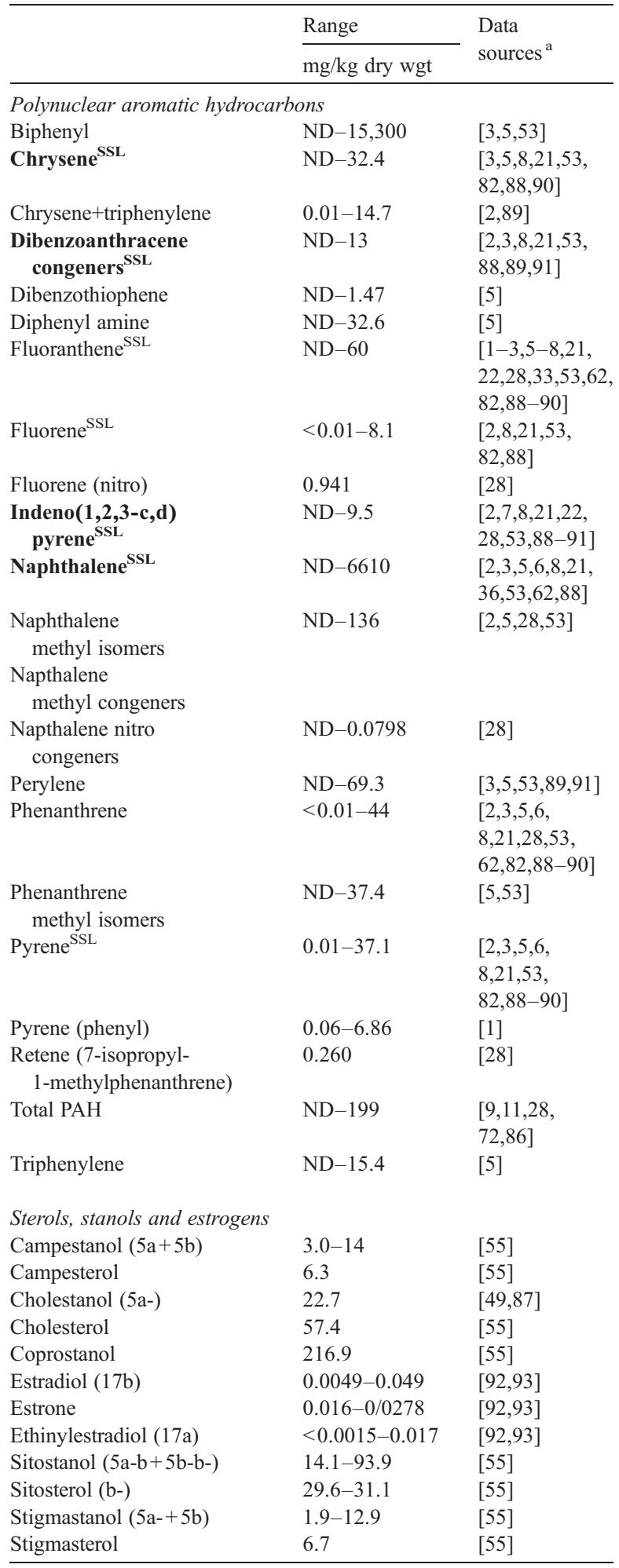

Table 1 (continued)

\begin{tabular}{|c|c|c|}
\hline & Range & \multirow{2}{*}{$\begin{array}{l}\text { Data } \\
\text { sources }^{a}\end{array}$} \\
\hline & $\mathrm{mg} / \mathrm{kg}$ dry wgt & \\
\hline \multicolumn{3}{|l|}{ Surfactants } \\
\hline Alcohol ethoxylates & ND-141 & {$[70,94,95]$} \\
\hline Alkylbenzene sulfonates & $<1-30,200$ & $\begin{array}{l}{[6,7,9,} \\
70-72,74, \\
85,94,96-98]\end{array}$ \\
\hline Alkylphenolcarboxylates & $10-14$ & {$[92]$} \\
\hline Alkylphenolethoxylates & ND-7214 & $\begin{array}{l}{[2,7,25,28,} \\
49,69,71,72, \\
85,90,92, \\
94,99-101]\end{array}$ \\
\hline $\begin{array}{l}\text { Alkyphenols (nonyl } \\
\text { and octylphenol) }\end{array}$ & ND-559,300 & $\begin{array}{l}{[2,6,9,18,25,} \\
28,36,49,64, \\
69,74,92, \\
95,99-107]\end{array}$ \\
\hline Coconut diethanol amides & $0.3-10.5$ & {$[70]$} \\
\hline Poly(ethylene glycol)s & $1.7-17.6$ & {$[70]$} \\
\hline \multicolumn{3}{|l|}{ Triaryl/alkyl phosphate esters } \\
\hline Cresyldiphenyl phosphate & $0.61-179$ & {$[3]$} \\
\hline Tricresyl phosphate & $0.069-1650$ & [3] \\
\hline Tricresyl phosphate & $<0.020-12.000$ & {$[2]$} \\
\hline Tri- $n$-butylphosphate & $<0.020-2.400$ & {$[2]$} \\
\hline Triphenylphosphate & $<0.020-1.900$ & {$[2]$} \\
\hline Trixylyl phosphate & $0.027-2420$ & {$[3]$} \\
\hline
\end{tabular}

See Supporting Information 1 for further detail.

Boldfaced $=$ one or more reported concentrations exceed an SSL. SSLs may be established only for a particular congener. Table 1 groups congeners and where any one of the congener concentration exceeds an SSL for that congener, the group of congeners is shown in bold. Available data for specific congeners is shown in supporting information 2 .

${ }^{\text {SSL }}$ indicates that SSLs have been established for one or more congener in this group.

ND indicates not detected where the lower limit of detection is not specified. $>X X$ indicates not detected at the specified $(X X)$ limit of detection.

${ }^{a}$ The data sources for this table are identified by number and cited below as a part of this table.

Data sources:

1. Jacobs LW, O'Connor GA, Overcash MA, Zabik MJ, Rygiewicz, P. Land application of sludge: food chain implications. In Effects of trace organics in sewage sludges on soil-plant systems and assessing their risk to humans, 1987.

2. Torslov J, Samsoe-Peterson L, Rasmussen, JO, Kristensen P. Use of waste products in agriculture: contamination level, environmental risk assessment and recommendations for quality criteria. VKI Institute for the Water Environment, 1997: Ministry of Environment and Energy Denmark, Danish EPA.

3. U.S. Environmental Protection Agency. An overview of the contaminants of concern in the disposal and utilization of municipal sewage sludge, 1983.

4. Katsoyiannis A, Samara C. Persistent organic pollutants (POPs) in the sewage treatment plant of Thessaloniki, northern Greece: occurrence and removal. Water Res, 2004, 38, 2685-2698.

5. U.S. Environmental Protection Agency. Technical support document for the round two sewage sludge pollutants. EPA-822-R-96-003, 
Notes to Table 1:

1996a, Office of Water, Office of Science and Technology, Health and Ecological Criteria Division: Washington.

6. Wild SR, Jones KC. Organic chemicals entering agricultural soils in sewage sludges: screening for their potential to transfer to crop plants and livestock. Sci Total Environ, 1992, 119, 85-119.

7. Drescher-Kaden U, Bruggemann R, Matthes B, Matthies M. Contents of organic pollutants in German sewage sludges, in Effects of organic contaminants in sewage sludge on soil fertility, plants and animals, J.E. Hall, D.R. Sauerbeck, and P. L'Hermite, Editors. 1992, Commission of the European Communities: Luxembourg.

8. Bright DA, Healey N. Contaminant risks from biosolids land application: contemporary organic contaminant levels in digested sewage sludge from five treatment plants in greater Vancouver, British Columbia. Environ Pollut, 2003, 126, 39-49.

9. Schnaak W, Kuchler T, Kujawa M, Henschel K-P, Subenbach D, Donau R. Organic contaminants in sewage sludge and their ecotoxicological significance in the agricultural utilization of sewage sludge. Chemosphere, 1997, 35, 5-11.

10. Nicholls CR, Allchin CR, Law RJ. Levels of short and medium chain length polychlorinated $n$-alkanes in environmental samples from selected industrial areas in England and Wales. Environ Pollut, 2001, 114, 415-430.

11. Frost P, Camenzind R, Magert A, Bonjour R, Karlaganis, G. Organic micropollutants in Swiss sewage sludge. J Chromatogr A, 1993, 643, 379-388.

12. Kulling D, Candinas T, Stadelmann FX. Nahrstoffe und Schwermetalle im Klarschlamm. Agrarforschung, 2002, 9, 200-205. 13. Chevreuil M, Granier L, Chesterikoff A, Letolle R. Polychlorinated biphenyls partitioning in waters from river, filtration plant and wastewater plant: the case for Paris (France). Water Res, 1990, 24, 1325-1333.

14. Fendinger NJ, McAvoy DC, Eckhoff WS, Price BB. Environmental occurrence of polydimethylsiloxane. Environ Sci Technol, 1997, $31,1555-1563$.

15. Batley GE, Hayes JW. Polyorganosiloxanes (Silicones) in the aquatic environment of the Sydney region. Aust J Mar Freshw Res, 1991, 42, 287-293.

16. Watanabe N, Yasuda $Y$, Kato $K$, Nakamura T, Funasada R, Shimokawa K, Sato E, Ose Y. Determination of trace amounts of siloxanes in water, sediments and fish tissues by inductively coupled plasma emission spectrometry. Sci Total Environ, 1984, 34, 169-176. 17. Watanabe N, Nakamura T, Watanabe E, Sato E, Ose Y. Distribution of organosiloxanes (silicones) in water, sediments and fish from the Nagara River watershed. Japan. Sci Total Environ, 1984, 35, 91-97. 18. Gehring M, Vogel D, Tennhardt L, Weltin D, Bilitewski B. Efficiency of sewage sludge treatment technologies at eliminating endocrine active compounds. Waste Management and the Environment II, 2004, 621-630.

19. Wang M-J, McGrath SP, Jones KC. Chlorobenzenes in field soil with a history of multiple sewage sludge applications. Environ Sci Technol, 1995, 29, 356-362.

20. Rogers HR, Campbell JA, Crathorne B, Dobbs, AJ. The occurrence of chlorobenzenes and permethrins in twelve U.K. sewage sludges. Water Res, 1989, 23, 913-921.

21. Berset JD, Etter-Holzer R. Quantitative determination of polycyclic aromatic hydrocarbons, polychlorinated biphenyls and organochlorine pesticides in sewage sludges using supercritical fluid extraction and mass spectrometric detection. J Chromatogr A, 1999, $852,545-558$.

22. Witte H, Langenohl T, Offenbacher, G. Investigation of the entry of organic pollutants into soils and plants through the use of sewage sludge in agriculture. Part A: organic pollutant load in sewage sludge. Korresp Abw, 1988, 13, 118-125.

23. North KD. Tracking polybrominated diphenl ether releases in a wastewater treatment plant effluent, Palo Alto, California. Environ Sci Technol, 2004, 38, 4484-4488.

24. Hale RC, La Guardia MJ, Harvey EP, Mainor TM. Potential role of fire retardant-treated polyurethane foam as a source of brominated diphenyl ethers to the US environment. Chemosphere, 2002, 46, 729-735.

25. La Guardia M, Hale RC, Harvey E, Bush EO, Mainor TM, Gaylor MO. Organic contaminants of emerging concern in land-applied sewage sludge (biosolids). J Res Sci Tech, 2004, 1, 111-122.

26. Hellstrom T. Brominated flame retardants (PBDE and PBB) in sludge-a problem? in The Swedish Water and Wastewater Association, 2000.

27. de Boer J, de Boer K, Boon, JP. Polybrominated biphenyls and diphenyl ethers, in The handbook of environmental chemistry. Paasivirta J, Editor. 2000, Springer: Berlin.

28. Vikelsoe J, Thomsen M, Carlsen L, Johansen E. Persistent organic pollutants in soil, sludge and sediment. NERI technical report no. 402. 2002, National Environment Research Institute. Ministry of the Environment. p. 98

29. Hale RC, LaGuardia MJ, Harvey EP, Gaylor MO, Mainor TM, Duff, WH. Persistent pollutants in land-applied sludges. Nature, 2001, 412, 140-141.

30. Litz, N. Some investigations into the behavior of pentabromodipheyl ether (PeBDE) in soils. J Plant Nutr Soil Sci, 2002, 165, 692696.

31. Morris S, Allchin CR, Zegers BN, Haftka JJH, Boon JP, Belpaire C, Leonards PEG, Leeuwen SPJV, De Boer, J. Distribution and fate of HBCD and TBBPA brominated flame retardants in North Sea estuaries and aquatic food webs. Environ Sci Technol, 2004, 38, 5497-5504.

32. Sellstrom, U, Jansson, B. Analysis of tetrabromobisphenol A in a product and environmental samples. Chemosphere, 1995, 31, 30853092 .

33. O'Connor, GA. Organic compounds in sludge-amended soils and their potential for uptake by crop plants. Sci Total Environ, 1996, 185, 71-81.

34. Klee N, Gustavsson L, Kosmehl T, Engell M. Changes in toxicity and genotoxicity of industrial sewage sludge samples containing nitroand animo-aromatic compounds following treatment in bioreactors with different oxygen regimes. Environ Sci Pollut Res Int, 2004, 11, 313-320.

35. Wilson SC, Alcock RE, Sewart AP, Jones KC. Organic chemicals in the environment: persistence of organic contaminants in sewage sludge-amended soil: a field experiment. J Environ Qual, 1997, 26, 1467-1477.

36. Kirchmann H, Tengsved A. Organic pollutants in sewage sludge Swedish J Agric Res, 1991, 21, 115-119.

37. Herren, D, Berset, JD. Nitro Musks, Nitro musk amino metabolites and polycyclic musks in sewage sludges: quantitative determination by HRGC-ion-trap-MS/MS and mass spectral characterization of the amino metabolites. Chemosphere 2000. 40, 565-574.

38. Mumma RO, Raupach DC, Waldman JP, Tong SSC, Jacobs ML, Babish JG, Hotchkiss JH, Wszolek PC, Gutenman WH, Bache CA, Lisk DJ. National survey of elements and other constituents in municipal sewage sludges. Arch Environ Contam Toxicol, 1984, 13, 75-83.

39. Chau YK, Zhang S, Maguire RJ. Occurrence of butyl in species in sewage and sludge in Canada. Sci Total Environ, 1992, 121, $271-281$. 
Notes to Table 1:

40. Fent K, Muller MD. Occurrence of organotins in municipal wastewater and sewage sludge and behavior in a treatment plant. Environ Sci Technol, 1991, 25, 489-493.

41. Fent K, Hunn J, Renggli D, Siegrist H-R. Fate of tributyltin in sewage sludge treatment. Mar Environ Res, 1991, 32, 223-231.

42. Fent K. Organotin speciation in municipal wastewater and sewage sludge: ecotoxicological consequences. Mar Environ Res, 1989, 28, 477-483.

43. Fent K. Organotin compounds in municipal wastewater and sewage sludge: contamination, fate in treatment process and ecotoxicological consequences. Sci Total Environ, 1996, 185, 151-159.

44. Voulvoulis N, Scrimshaw MD, Lester JN. Removal of organotins during sewage treatment: a case study. Environ Technol, 2004, 25, 733-740.

45. Khan SJ, Ongerth JE. Estimation of pharmaceutical residues in primary and secondary sewage sludge based on quantities of use and fugacity modelling. Water Sci Technol, 2002, 46, 105-113.

46. Golet EM, Strehler A, Alder AC, Giger W. Determination of fluoroquinolone antibacterial agents in sewage sludge and sludgetreated soil using accelerated solvent extraction followed by solidphase extraction. Anal Chem, 2002, 74, 5455-5462.

47. Lindberg RH, Wennberg P, Tysklind M, Andersson BAV. Screening of human antibiotic substances and determination of weekly mass flows in five sewage treatment plants in Sweden. Environ Sci Technol, 2005, 39, 3421-3429.

48. McAvoy DC, Schatowitz B, Jacob M, Hauk A, Eckhoff WS. Measurement of triclosan in wastewater treatment systems. Environ Toxicol Chem, 2002, 21, 1323-1329.

49. Lee HB, Peart TE. Organic contaminants in Canadian municipal sewage sludge. Part I. Toxic or endocrine-disrupting phenolic compounds. Water Qual Res J Can, 2002, 37, 681-696.

50. Bester K. Triclosan in a sewage treatment process - balances and monitoring data. Water Res, 2003, 37, 3891-3896.

51. Poiger T, Field JA, Field TM, Siegrist H, Giger W. Behavior of fluorescent whitening agents during sewage treatment. Water Res 1998. 32, 1939-1947.

52. Difrancesco AM, Chiu PC, Standley LJ, Allen HE, Salvito DT. Dissipation of fragrance materials in sludge-amended soils. Environ Sci Technol, 2004, 38, 194-201.

53. Stevens JL, Northcott GL, Stern GA, Tomy GT, Jones KC. PAHs, PCBs, PCNs, organochlorine pesticides, synthetic musks, and polychlorinated $n$-alkanes in U.K. sewage sludge: survey results and implications. Environ Sci Technol, 2003, 37, 462-467.

54. Kupper T, Berset JD, Etter-Holzer R, Furrer R, Tarradellas J. Concentrations and specific loads of polycyclic musks in sewage sludge originating from a monitoring network in Switzerland. Chemosphere, 2004, 54, 1111-1120.

55. Balk F, Ford RA. Environmental risk assessment for the polycyclic musks, AHTN and HHCB in the EU I. Fate and exposure assessment. Toxicology Letters, 1999a, 111, 57-79.

56. Bester K. Retention characteristics and balance assessment for two polycyclic musk fragrances (HHCB and AHTN) in a typical German sewage treatment plant. Chemosphere, 2004, 57, 863-870.

57. Berset JD, Bigler P, Herren D. Analysis of nitro musk compounds and their amino metabolites in liquid sewage sludges using NMR and mass spectrometry. Anal Chem, 2000, 72, 2124-2131.

58. U.S. Environmental Protection Agency. National sewage sludge survey; availability of information and data, and anticipated impacts on proposed regulations; proposed rule. Part III. Federal Register, 1990, $55,47210-47283$.
59. McIntyre, AE, Lester JN. Occurrence and distribution of persistent organochlorine compounds in U.K. sewage sludge. Water Air Soil Pollut 1984. 23, 397-415.

60. McIntyre AE, Lester JN. Polychlorinated biphenyl and organochlorine insecticide concentrations in forty sewage sludges in England. Environ Pollut Series B, 1982, 3, 225-230.

61. Kirk PWW, Lester JN. The behaviour of chlorinated organics during activated sludge treatment and anaerobic digestion. Water Sci Technol, 1988, 20, 353-359.

62. Ahmad UK, Ujang Z, Woon $\mathrm{CH}$, Indran $\mathrm{S}$, Mian $\mathrm{MN}$. Development of extraction procedures for the analysis of polycyclic aromatic hydrocarbons and organochlorine pesticides in municipal sewage sludge. Water Sci Technol, 2004, 50, 137-144.

63. Woodhead, D. Permethrin trials in the Meltham sewage catchment area. Water Services, 1983, 87, 198-202.

64. Bolz U, Hagenmaier H, Korner W. Phenolic xenoestrogens in surface water, sediments, and sewage sludge from BadenWurttemberg, south-west Germany. Environ Pollut Series A, 2001, 115, 291-301.

65. Lee H-B, Peart TE. Bisphenol A contamination in Canadian municipal and industrial wastewater and sludge samples. Water Qual Res J Can 2000, 35, 283-298.

66. DeWalle FB, Kalman DA, Dills R, Norman D, Chian ESK, Giabbai M, Ghosal M. Presence of phenolic compounds in sewage, effluent and sludge from municipal sewage treatment plants. Water Sci Technol, 1982, 14, 143-150.

67. Ettala M, Koskela J, Kiesila A. Removal of chlorophenols in a municipal sewage treatment plant using activated sludge. Water Sci Technol, 1992, 26, 797-804.

68. Wild SR, Harrad SJ, Jones KC. Chlorophenols in digested U.K. sewage sludges. Water Res 1993, 27, 1527-1534.

69. Vikelsoe J, Thomsen M, Carlsen L. Phthalates and nonylphenols in profiles of differently dressed soils. Sci Total Environ, 2002, 296, 105-116.

70. Petrovic M, Barcelo D. Determination of anionic and nonionic surfactants, their degradation products, and endocrine-disrupting compounds in sewage sludge by liquid chromatography/mass spectrometry. Anal Chem, 2000, 72, 4560-4567.

71. Langenkamp H, Part P, Erhardt W, Prueb A. Organic contaminants in sewage sludge for agricultural use. European Commission and UMEG Center for Environmental Measurements. Environmental Inventories and Product Safety, 2001.

72. Paulsrud B, Wien A, Nedland KT. A survey of toxic organics in Norwegian sewage sludge, compost, and manure. in 8th Annual International Conference of the FAO ESCORENA Network of Recycling of Agricultural, Municipal and Industrial Residues in Agriculture (Formerly Animal Waste Management), May 26-28, 1998. Rennes, France.

73. Berset JD, Etter-Holzer R. Determination of phthalates in crude extracts of sewage sludges by high-resolution capillary gas chromatography with mass spectrometric detection. J AOAC Int, 2001, 84, 383-391.

74. Langenkamp H. Workshop on Problems Around Sludge, ed. Marmo L, 1999: European Commission: Joint Research Center. 84. 75. U.S. Environmental Protection Agency, Standards for the use or disposal of sewage sludge: decision not to regulate dioxins in landapplied sewage sludge. Federal Register 68(206) 61083-61096, 2003.

76. Stevens JL, Jones KC. Quantification of PCDD/F concentrations in animal manure and comparison of the effects of the application of cattle manure and sewage sludge to agricultural land on human exposure to PCDD/Fs. Chemosphere, 2003, 50, 1183-1191. 
Notes to Table 1:

77. Wild SR, Harrad SJ, Jones KC. The influence of sewage sludge applications to agricultural land on human exposure to polychlorinated dibenzo-p-dioxins (PCDDs) and -furans (PCDFs). Environ Pollut 1994, 83, 357-369.

78. Ho A, Clement RE. Chlorinated dioxins/furans in sewage and sludge of municipal water pollution control plants. Chemosphere, 1990, 20, 1549-1552.

79. Eljarrat E, Caixach J, Rivera J. A comparison of TEQ contributions from PCDDS, PCDFs, and dioxin-like PCBs in sewage sludges from Catalonia, Spain. Chemosphere, 2003, 51, 595-601.

80. Van oostdam JC, Ward JEH. Dioxins and furans in the British Columbia environment, 1995. Environmental Protection Department, Victoria, British Columbia.

81. Alvarado MJ, Armstrong S, Crouch E. The AMSA 2000/2001 survey of dioxin-like compounds in biosolids: statistical analyses. 2001. Cambridge Environmental: Cambridge, MA.

82. Lazzari L, Sperni L, Bertin P, Pavoni B. Correlation between inorganic (heavy metals) and organic (PCBs and PAHs) micropollutant concentrations during sewage sludge composting processes. Chemosphere, 2000, 41, 427-435.

83. Blanchard M, Teil MJ, Ollivon D, Legenti L, Chevreuil M. Polycyclic aromatic hydrocarbons and polychlorobiphenyls in wastewaters and sewage sludges from the Paris area (France). Environ Res, 2004, 95, 184-197.

84. Berset JD, Etter-Holzer R. Determination of coplanar and ortho substituted PCBs in some sewage sludges of Switzerland using HRGC/ECD and HRGC/MSD. Chemosphere, 1996, 32, 2317-2333. 85. Marcomini A, Capel PD, Capel PD, Hani H. Residues of detergentderived organic pollutants and polychlorinateed biphenyls in sludgeamended soil. Naturwissenschaften, 1988, 75, 460-462.

86. Blanchard M, Teil M-J, Ollivon D, Garban B, Chestérikoff C, Chevreuil M. Origin and distribution of polyaromatic hydrocarbons and polychlorobiphenyls in urban effluents to wastewater treatment plants of the Paris area (France). Water Res, 2001, 35, 3679-3687.

87. Balzer W, Pluschke P. Secondary formation of PCDD/F during the thermal stabilization of sewage sludge. Chemosphere, 1994, 29, 1889-1902.

88. Perez S, Guillamon M, Barcelo D. Quantitative analysis of polycyclic aromatic hydrocarbons in sewage sludge from wastewater treatment plants. J Chromatogr A, 2001, 938, 57-65.

89. Bodzek D, Janoszka B. Comparison of polycyclic aromatic compounds and heavy metals contents in sewage sludges from industrialized and non-industrialized region. Water Air Soil Pollut, 1999, 111, 359-369.

90. Oleszczuk P, Baran S. The concentration of mild-extracted polycyclic aromatic hydrocarbons in sewage sludges. J. Environ. Sci. Health Part A Toxic-Hazard. Subst Environ Eng, 2004, 39, 2799-2815.

91. Harms H, Sauerbeck DR. Toxic organic compounds in town waste materials: their origin, concentration and turnover in waste composts, soils, and plants. in Environmental effects of organic and inorganic contaminants in sewage sludge, 1982. Stevenage, UK: D. Reidel Publishing Company, England.

92. Ternes TA, Andersen H, Gilberg D, Bonerz M. Determination of estrogens in sludge and sediments by liquid extraction and GC/MS/ MS. Chemistry, 2002, 74, 3498-3504.
93. Andersen H, Siegrist H, Halling-Sorensen B, Ternes TA. Fate of estrogens in municipal sewage treatment plant. Environ Sci Technol, 2003, 37, 4021-4026.

94. Cavalli L, Valtorta L. Surfactants in sludge-amended soil. Tenside Surf Det, 1999, 36, 22-28.

95. Cantero M, Rubio S, Perez-Bendito, D. Determination of non-ionic polyethoxylated surfactants in sewage sludge by coacervative extraction and ion trap liquid chromatography mass spectrometry. $\mathrm{J}$ Chromatogr A, 2004, 1046, 147-153.

96. Field JA, Miller DJ, Field TM, Hawthorne SB, Giger W. Quantitative determination of sulfonated aliphatic and aromatic surfactants in sewage sludge by ion-pair/supercritical fluid extraction and derivation gas chromatography/mass spectrometry. Anal Chem, 1992, 64, 3161-3167.

97. Prats D, Rutz F, Vazquez B, Zarzo D, Berna JL, Moreno A. LAS homolog distribution shift during wastewater treatment and composting: ecological implications. Environ Toxicol Chem, 1993, 12, 1599-1608.

98. Feijtel TCJ, Rottiers A, Rijs GBJ, Kiewiet A, deNijs, A. AIS/ CESIO environmental surfactant monitoring programme. Part 1. LAS monitoring study in "De Meern" sewage treatment plant and receiving river "Leidsche Rijn." Chemosphere, 1995, 30, 1053-1066.

99. Marcomini A, Capel PD, Lichtensteiger T, Brunner PH, Giger W. Behavior of aromatic surfactants and PCBs in sludge-treated soil and landfills. J Environ Qual, 1989, 18, 523-528.

100. Keller H, Xia K, Bhandari A. Occurrence and degradation of estrogenic nonylphenol and its precursors in northeast Kansas wastewater treatment plants. Prac Per of Haz, Tox and Radio Waste Man, 2003, 7, 203-213.

101. La Guardia MJ, Hale RC, Harvey E, Mainor TM. Alkylphenol ethoxylate degradation products in land-applied sewage sludge (biosolids). Environ Sci Technol, 2001, 35, 4798-4804.

102. Bennett ER, Metcalfe CD. Distribution of alkylphenol compounds in Great Lakes sediments, United States and Canada. Environ Toxicol Chem, 1998, 17, 1230-1235.

103. Lee HB, Peart TE. Determination of 4-nonylphenol in effluent and sludge from sewage treatment plants. Anal Chem, 1995, 67, 1976-1980.

104. Pryor SW, Hay AG, Walker LP. Nonylphenol in anaerobically digested sewage sludge from New York State. Environ Sci Technol, 2002, 36, 3678-3682.

105. Bennie, DT. Review of the environmental occurrence of alkylphenols and alkylphenol ethoxylates. Water Qual Res J Can, 1999, 34, 79-122.

106. Jobst H. Chlorophenols and nonylphenols in sewage sludges. Part II: did contents of pentachlorophenol and nonylphenols reduce? Acta Hydrochim. Hydrobiol, 1998, 26, 344-348.

107. Xia, K, Pillar, G. Anthropogenic organic chemicals in biosolids from selected wastewater treatment plants in Georgia and South Carolina, April 23-24. In Proceedings of the 2003 Georgia Water Resources Conference, 2003. Athens, Georgia. 
by risk-based analyses. The Ontario regulatory maximum soil concentration limits address several different land uses and pathways of exposure for 118 chemicals (Ontario Ministry of the Environment, 2004). The Dutch system includes target values that seek to prevent harm to human and ecological systems as well as intervention values where predicted harm requires clean-up to be implemented. The Ontario and Dutch values are generally comparable to the U.S. SSLs, but values for specific chemicals are not identical, presumably due to differences in assumptions (Netherlands Ministry of Housing Spatial Planning and Environment, 2000).

For the purposes of this paper, we compared the reported sludge concentrations to the SSL values for those compounds for which EPA has established an SSL. The SSLs are not regulatory standards, but are guidelines used by EPA relative to cleaning up industrially-contaminated sites. Sites with soil concentrations lower than the SSLs are considered "clean," while sites with higher concentrations require sitespecific risk analysis. Using default values for a residential exposure scenario, the EPA risk-based SSLs address exposure pathways including direct ingestion of contaminated soil, inhalation, dermal exposure, drinking of groundwater contaminated by migration of chemicals through soil, and ingestion of homegrown produce contaminated via plant uptake (U. S. Environmental Protection Agency Superfund, 1996). The groundwater pathway includes two values, one assuming no dilution or attenuation (1 DAF) and the other assuming a 20-fold dilution/attenuation (20 DAF). SSLs do not include risks posed by ingesting animal products grown on contaminated soils, nor do they address environmental and ecologic risks. These human health SSLs are based on a $10^{-6}$ risk for carcinogens or a hazard quotient of 1 for non-carcinogens and separate SSL concentrations are listed for four different exposure pathways (ingestion, inhalation, groundwater $20 \mathrm{DAF}$, groundwater 1 DAF). For most organic contaminants, the groundwater SSL that assumes no attenuation or dilution is the most restrictive concentration (supporting information 2).

It is likely that the concentration of a chemical in a soil to which sludge has been applied would be lower than the concentration in the sludge itself due to mixing and subsequent dilution with soil as well as through degradation, volatilization and leaching processes. A single application of sludge tilled into the soil would be diluted approximately 100 -fold, but concentrations would increase with repeated applications when losses are not as great as application rates and would also be higher in surface soils if sludge is not tilled into the soil such as in pasture application. Despite the differences between contaminated soils and sludges, the NRC (National Research Council, 2002) used SSLs as an EPA-established metric to suggest whether further evaluation might be warranted. We thus report sludge concentrations of organic contaminants that exceed an SSL (Table 1; supporting information 2).

Two other EPA-generated lists of chemicals were also used to evaluate the organic chemicals reported in sludges. The first is a list of chemicals generated in 1979 and modified in 1981 for which technology-based water effluent limitations were required (Keith and Telliard, 1979). These 126 chemicals, known as priority pollutants, reflect the knowledge of contaminants in industrial wastewater effluents during the 1970s. One hundred and eleven of these are organic chemicals. Although there are no federal requirements for monitoring these compounds in sewage sludges, some states, including New York (New York State Department of Environmental Conservation, 2003), require screening of land-applied sludges for these priority pollutants. The second list includes chemicals that laboratories performing analyses on Superfund site soils must be able to detect and quantify. These 143 chemicals are known as target compounds (U.S. Environmental Protection Agency, 2004). Table 2 provides a summary, by class, of the number of chemicals reported in sludges that fall into these groups.

\section{Results and discussion}

Tens of thousands of organic chemicals are currently in use, however sludge concentration data could only be found for 516 organic chemicals in the peer reviewed literature and official government reports (supporting information 1). Table 2 shows the number of compounds in each of the 15 classes for which concentration data were found, and the number of studies from which these data were obtained.

Ninety of the 111 organic priority pollutants and 101 of the 143 target compounds were reported in sludges (Table 2). No data were found for the other 21 organic priority pollutants or 42 target compounds. Eighty-three percent of the reported chemicals were not on the priority pollutant list and $80 \%$ were not on the target compounds list. Thus monitoring sludges for priority pollutants will not capture the vast majority of chemicals that may be present.

Six of the 15 chemical classes for which data were found did not contain compounds included among the priority pollutants, target compounds, or those compounds with SSLs (Table 2). This may be due in part to 
Table 2

Number of chemicals reported in sludges in each class, number of studies from which data were obtained, number that are priority pollutants, target compounds or for which there are SSLs, and number for which maximum reported concentrations in sludges exceed an SSL

\begin{tabular}{|c|c|c|c|c|c|c|}
\hline & \# chem & \# of studies & \# PP chem & \# TC chem & \# chem with SSLs & \# chem that exceed an SSL \\
\hline Aliphatics & 58 & 19 & 16 & 17 & 16 & 15 \\
\hline Chlorobenzenes & 11 & 13 & 6 & 7 & 5 & 5 \\
\hline Flame retardants & 29 & 11 & 0 & 0 & 0 & \\
\hline Monocyclic HC & 34 & 12 & 7 & 12 & 11 & 10 \\
\hline Nitrosamines & 7 & 1 & 2 & 1 & 1 & 1 \\
\hline Organotins & 6 & 7 & 0 & 0 & 0 & \\
\hline PCPs & 36 & 17 & 0 & 0 & 0 & \\
\hline Pesticides & 71 & 20 & 18 & 19 & 18 & 15 \\
\hline Phenols & 40 & 20 & 10 & 14 & 9 & 8 \\
\hline Phthalate & 19 & 16 & 9 & 8 & 6 & 6 \\
\hline PCBs & 108 & 38 & 5 & 6 & 0 & \\
\hline PAHs & 52 & 25 & 18 & 18 & 13 & 8 \\
\hline Sterols and stanols & 16 & 3 & 0 & 0 & 0 & \\
\hline Surfactants & 23 & 33 & 0 & 0 & 0 & \\
\hline Triaryl/alkyl phosphate.esters & 6 & 2 & 0 & 0 & 0 & \\
\hline Total & 516 & $113^{\mathrm{a}}$ & 91 & 102 & 79 & 68 \\
\hline
\end{tabular}

${ }^{\text {a }}$ Note: \# of studies is not a sum of the list above because some studies include data for more than one class.

the fact that all three of these lists arose out of a response to a concern over the fate of industrial contaminants. Thus some chemicals, such as personal care products, that are present in sludges primarily as a result of nonindustrial sources, do not appear on those lists. In addition, the priority pollutant list is 25 years old, so industrial chemicals of current and emerging concern, such as polybrominated diphenyl ethers, which were not in wide use at that time, were not included.

There are SSLs for $15 \%$ of the 516 organic chemicals reported in sludges. The reported maximum sludge concentration exceeded an SSL for $86 \%$ of the chemicals for which there are SSLs (Table 2, supporting information 2). This high proportion is observed in most classes, with PAHs as an exception.

The proportion of individual reports that exceed an SSL for a particular chemical was examined to determine whether such exceedances were the result of single high-concentration reports or whether most reported values exceeded an SSL. The data show that for chemicals in some classes such as aliphatics and monocyclic hydrocarbons, most reported concentrations for chemicals within that class exceed an SSL while for other classes including phthalates and polyaromatic hydrocarbons, a much smaller percentage of the reported concentrations were high enough to exceed an SSL (Table 3). However, even within these classes, there are some chemicals for which a high percentage of reports exceed an SSL (Fig. 1).

As a result of an evaluation of additional sludgeborne chemicals for which regulation should be considered, the EPA has suggested that it will conduct limited additional sludge testing including efforts to monitor the presence of 9 organic chemicals (acetone, anthracene, carbon disulfide, 4-chloroaniline, diazinon, fluoranthene, methyl ethyl ketone, phenol, and pyrene) (U.S. Environmental Protection Agency, 2003b). In the present work, no data were found for two of the 9

Table 3

The percentage of reported concentrations that exceed an SSL for chemicals within a class for which there are SSLs

\begin{tabular}{|c|c|c|c|c|c|}
\hline & $\begin{array}{l}\% \text { for which } 100 \% \\
\text { reports exceed SSL }\end{array}$ & $\begin{array}{l}\% \text { for which } 75-99 \% \\
\text { reports exceed }\end{array}$ & $\begin{array}{l}\% \text { for which } 50-74 \% \\
\text { reports exceed }\end{array}$ & $\begin{array}{l}\% \text { for which } 25-50 \% \\
\text { reports exceed }\end{array}$ & $\begin{array}{l}\% \text { for which } 0-25 \% \\
\text { reports exceed }\end{array}$ \\
\hline Aliphatics & 75 & 6 & 19 & 0 & 0 \\
\hline Chlorobenzenes & 20 & 20 & 60 & 0 & 0 \\
\hline Monocyclic & 75 & 8 & 0 & 0 & 17 \\
\hline Nitrosamines & 100 & & & & \\
\hline Pesticides & 31 & 13 & 25 & 6 & 19 \\
\hline Phenols & 22 & 22 & 33 & 11 & 11 \\
\hline Phthalate & 17 & 0 & 17 & 17 & 50 \\
\hline PAHs & 0 & 23 & 8 & 15 & 54 \\
\hline
\end{tabular}

See Supporting Information 2 for the specific chemicals and SSLs. 


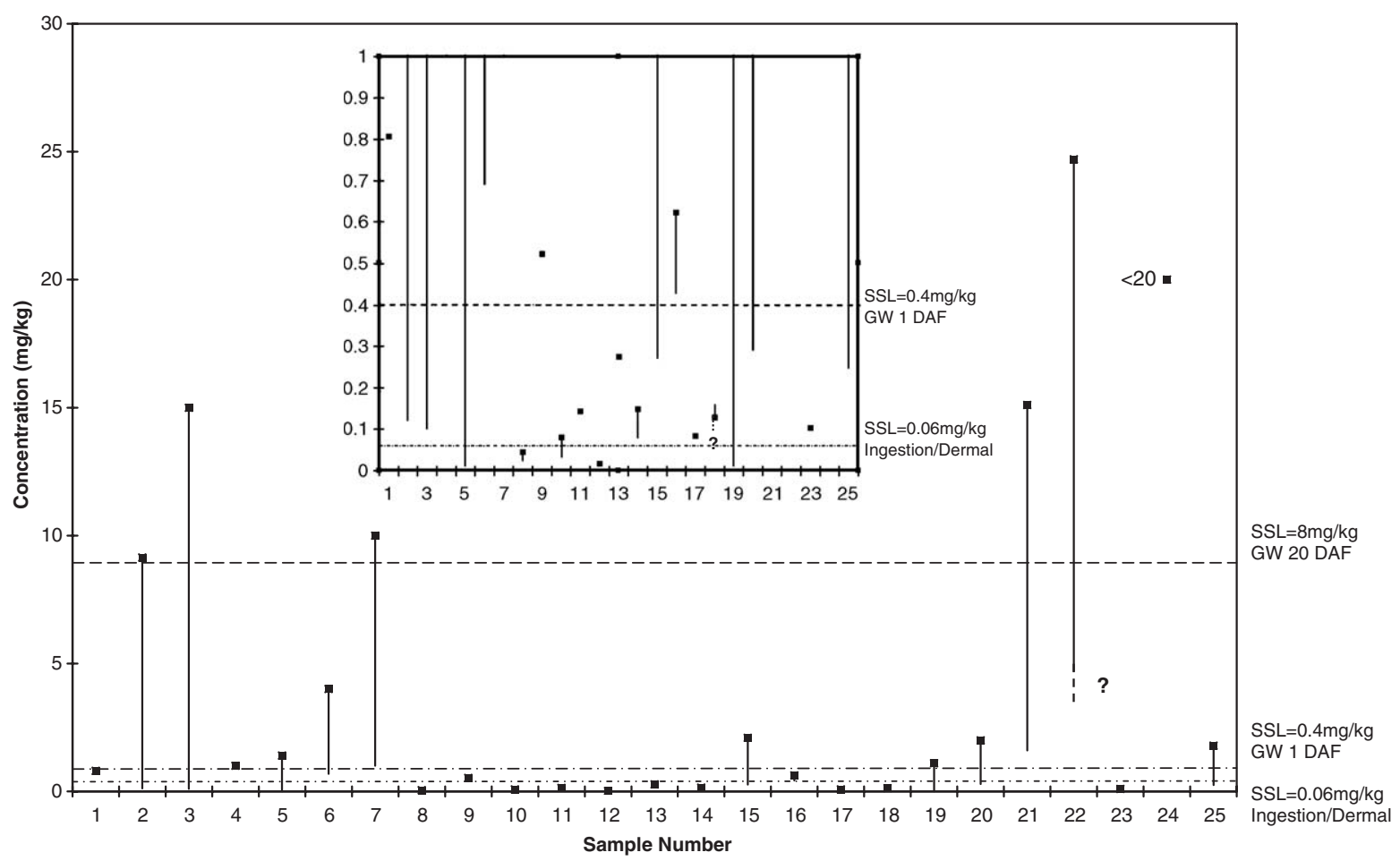

Fig. 1. Concentration (dry wgt) of benzo[a]pyrene in sewage sludges compared to soil screening levels. Note: ? means the report did not specify the concentration of values reported as non-detects.

compounds (acetone and methyl ethyl ketone). Data were found for the other 7 compounds (Table 1; supporting information 1; supporting information 2).

Anthracene was reported in 12 studies with a range from 0.0088 to $44 \mathrm{mg} / \mathrm{kg}$. Six studies detected more than $1 \mathrm{mg} / \mathrm{kg}$, but none exceeded an SSL. Only the NSSS reported concentrations for carbon disulfide, $p$-chloroaniline and diazinon, with maximum concentrations of $23.5,40.2$ and $0.15 \mathrm{mg} / \mathrm{kg}$ respectively. The carbon disulfide value exceeded the lower groundwater SSL and the $p$-chloroaniline value greatly exceeded both groundwater SSLs. There are no SSLs for diazinon. Fluoranthene was reported in 17 studies with concentrations ranging from 0.01 to $60 \mathrm{mg} / \mathrm{kg}$, but none exceeded any SSL. Seven studies reported phenol ranging from 0.002 to $920 \mathrm{mg} / \mathrm{kg}$, with concentrations of over $100 \mathrm{mg} / \mathrm{kg}$ reported in four studies, suggesting that these high concentrations were not a result of a particular source of contamination or analytic error. Six studies reported concentrations exceeding the lower groundwater SSL and four exceeded both groundwater SSLs. Eleven studies reported pyrene concentrations ranging from 0.1 to $36.8 \mathrm{mg} / \mathrm{kg}$, but none exceeded any SSL. These data suggest that several of the contaminants that EPA proposes to study are not likely to be of concern since data on their concentration in sludges exist and demonstrate concentrations below SSLs indicating they are unlikely to be present in concentrations high enough to be of significant risk.

Benzo(a)pyrene and hexachlorobenzene were suggested as pollutants requiring further analysis by the NRC in a 1996 report (National Research Council, 1996). In the present work, 19 sources reported benzo(a)pyrene in sludges at concentrations from $<0.01$ to $25 \mathrm{mg} / \mathrm{kg}$, with 24 of 27 reported concentrations exceeding one or more SSL (Fig. 1; supporting information 2). Hexachlorobenzene was reported by 9 sources. Nine of 13 reported concentrations exceed an SSL (Fig. 2; supporting information 2). These data suggest the value of assessing the risks posed by these chemicals in sludges.

Another group of compounds suggested as a possible concern is nitrosamines. Given the toxicity of nitrosamines and the potential for their formation during the wastewater treatment process, it is surprising that only two sources from the 1980s report nitrosamine concentration in sludges. Of the 7 compounds reported, there are SSLs for only one and the reported concentrations for that compound ( $N$-nitrosdiphenylamine) exceed the groundwater and ingestion/dermal SSLs. The NSSS detected $N$-Nitrosodiphenylamine in $1 \%$ of the sludges tested and hence it was eliminated from regulatory consideration by EPA. The maximum concentration 


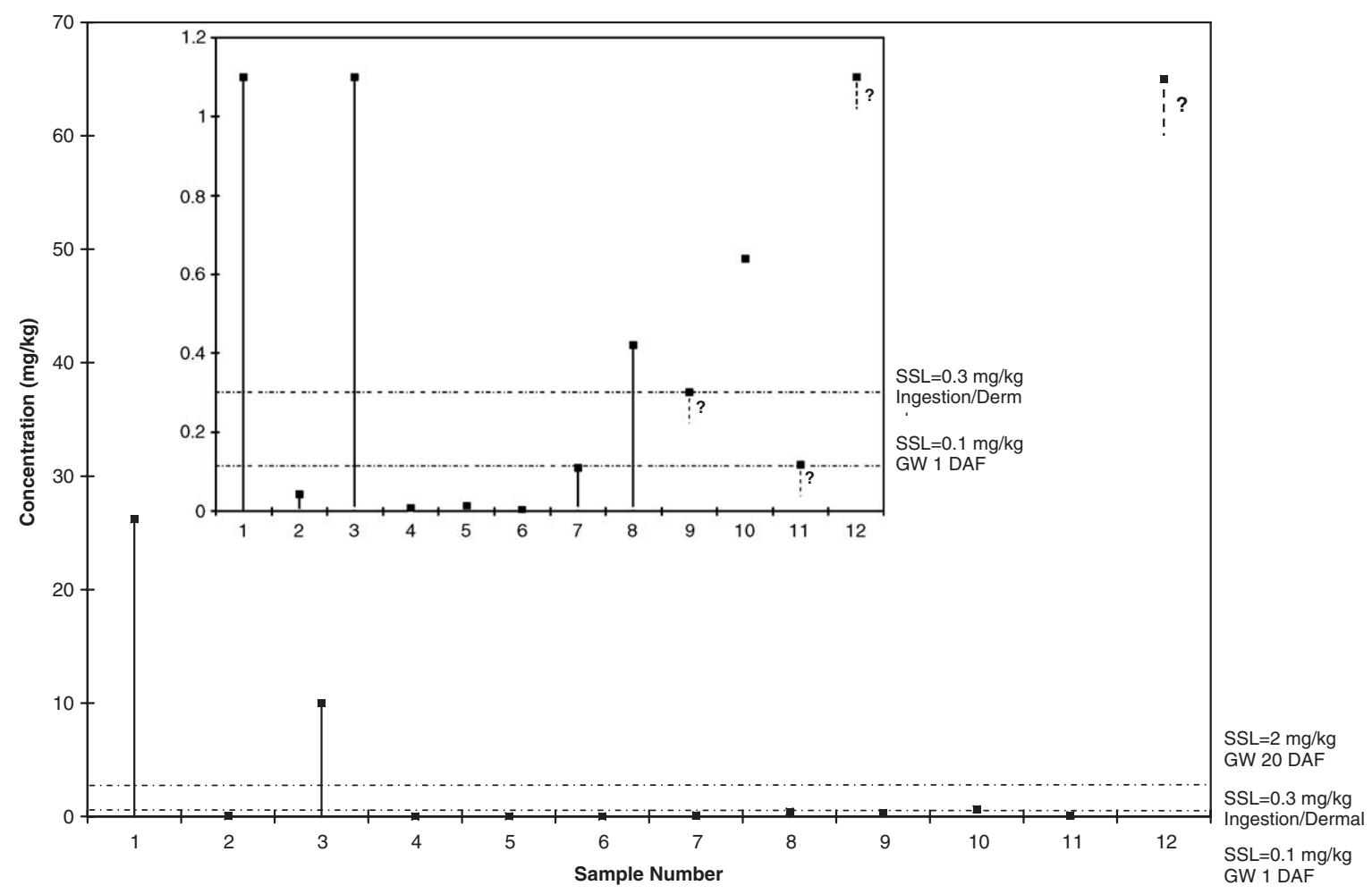

Fig. 2. Concentration (dry wgt) of hexachlorobenzene in sewage sludges compared to soil screening levels. Note: ? means the report did not specify the concentration of values reported as non-detects.

detected was $19.7 \mathrm{mg} / \mathrm{kg}$. Most samples had a limit of detection exceeding $1 \mathrm{mg} / \mathrm{kg}$ although detection limits as high as $800 \mathrm{mg} / \mathrm{kg}$ were also reported. The high limits of detection in many cases helped prompt the NRC to speculate that $N$-Nitrosodimethylamine may be present in some sludges at concentrations of concern (National Research Council, 1996).

Reported concentrations exceeding an SSL should not be interpreted to indicate a significant risk, but rather indicate that the concentration of those chemicals would be sufficient to require further assessment if present in soil at the same level. While sludge management and environmental processes may alter the concentrations of these chemicals in field situations through mixing with soil, leaching, degradation and other processes, the number of SSL exceedences suggests that assessment of the potential risks may be warranted.

The use of SSLs as a screening tool, does not address some potential routes of human exposure that may represent significant risk (Wild and Jones, 1992), including food chain transfer through the consumption of animal products. For organic contaminants in land applied sludges, this has been suggested as one of the two exposure pathways representing the highest risk, the other being direct ingestion of soil and sludge by humans
(Chaney et al., 1996). Application of sludge products to lawns, athletic fields and home gardens could provide a route for direct ingestion. The lipophilic nature of many organic chemicals found in sludges causes them to accumulate in the fat of exposed animals. Livestock may be exposed to sludge contaminants through sludge adhering to plant materials as well as through the ingestion of soil when sludges are applied to pasture (Fries, 1996).

Much of the work evaluating the potential risks posed by organic chemicals in sludges addresses human health risks. However, in addition to potential human impacts, organic chemicals in land applied sludges may pose environmental or ecological risks. The use of SSLs as a trigger does not account for these risks as most SSLs are currently based only on human health criteria. A number of the chemicals detected in sludges have been shown to function as endocrine disrupters. For example, nonylphenols which are present in sludges at relatively high concentrations (concentrations greater than $1000 \mathrm{mg} / \mathrm{kg}$ are not unusual), may be of concern because of their potential impact on wildlife (Environment Canada, 2004), even though they are unlikely to represent a major direct human health risk. Soil processes may also be impacted by organic chemical 
contaminants in land applied sludges as suggested by observed fungitoxic effects (Schnaak et al., 1997).

Specifying organic chemicals that should be monitored in sludges is not a simple task because it necessitates a degree of analytical competence that may not be widespread. The EPA has addressed this issue with respect to Superfund sites by developing a list of target compounds which includes priority pollutants in addition to other compounds. Certified laboratories performing analyses of Superfund samples are required to be able to test for these target compounds. As mentioned above, $80 \%$ of the organic chemicals reported in this paper, however, were not target compounds and could go undetected even in certified laboratories unless expensive mass spectral analyses were also performed. While the use of standardized methods that have been validated for individual chemicals is essential to ensure data quality, ongoing screening and validation efforts using generalized methods and robust detection technologies are required in order to identify chemicals of emerging concern.

For many compounds, there was wide variation in the reported concentrations found in sewage sludges. There are a number of potential sources of this variation. Discrepancies in analytical methods may account for some of the differences in the range of concentrations reported in this paper (Pryor et al., 2002). For most of the chemicals, no standard methods have been established for either sample extraction or analyte detection. The importance of methodological variation was clearly demonstrated in one report examining extraction efficiency, where a nearly five-fold difference was found in the concentration of several organic chemicals in sludge samples simply as a result of using different solvents (Bolz et al., 2001) and in another report where drying methods resulted in similarly large differences (Scrimshaw et al., 2004).

For some contaminants, differences in the source inputs to the WWTP may explain the range (Bodzek and Janoszka, 1999). For example, the high concentrations reported for some of the polynuclear aromatic hydrocarbons (PAHs) in one study (Constable et al., 1986) were likely due to inputs from local industry including two steel mills. Due to the large number of sludges sampled in the NSSS, that survey included a wide range of concentrations and yielded the highest reported concentrations for a number of contaminants (supporting information 1).

Another source of variability in chemical concentrations may be the type of treatment to which the sludges were subjected. The impact of this variable was difficult to gauge, however, as many reports did not provide information about wastewater and sludge processing methods. Where such information was available, it was noted (supporting information 1). Since pollutant con- centrations have been found to vary significantly with different types of processing (Wild and Jones, 1989), some of the variation in concentrations may have been a result of the different treatments to which the sludges were subjected (Constable et al., 1986; Wild and Jones, 1989; Zitomer and Speece, 1993; Rogers, 1996) or to differences in sludge retention time (Ternes et al., 2004).

Changes in chemical use over time is another potential source of the large range in reported concentrations. The references from which data were obtained go back as far as 1976, though most were from the 1980s or later. Because of changes in chemical usage, including bans on some chemicals, the introduction of new chemicals and the increasing use of others, the use of old data can be problematic. A new survey of organic chemicals in sludges is needed since the NSSS dates back to 1988 (National Research Council, 2002). Due to the paucity of data, however, even older studies were included in this paper and the date of sampling was included when available (supporting information 1).

The vast majority of the data found were for sludges from the U.S. or Western Europe where chemical use and wastewater treatment are relatively similar, resulting in similar pollutant concentrations. There were, however, some noteworthy differences. In several European countries, for example, bans or the voluntary elimination of compounds such as penta-brominated diphenyl ethers and nonylphenol have been enacted. As a result, concentrations of these chemicals in sludges from those countries have decreased in recent years (Jobst, 1998).

There are also important differences between the European and U.S. approaches to the management of land application of sludges that would likely result in lower soil loadings of contaminants in most European countries. The soil concentration of a sludge-borne pollutant after land application is not only a function of the concentration of the chemical in the sludge, but also the amount of sludge applied. A number of European countries limit application rates either through direct limits on the number of dry MT/ ha/yr or by limiting application to $P$-based agronomic rates, which are far more restrictive than the $N$-based rates used in the U.S. In Denmark, for example, no more than $30 \mathrm{~kg} / \mathrm{ha} / \mathrm{yr}$ of P can be applied (Ministry of Environment and Energy, 1997). This equates to an application rate of approximately 1 dry MT/ha/yr. While quantitative limits vary among the European countries, most limit application to a maximum of 1-4 dry MT/ha/yr (Schowanek et al., 2004). In conducting risk assessments, the European Commission assumes an application rate of 5 dry MT/ha/ yr (European Commission Joint Research Centre, 2003). This compares to 10 dry MT/ha/yr which was the assumed high-end application rate used by EPA in developing the 
regulations for land application (U.S. Environmental Protection Agency, 1995). Another critical management strategy pertains to the prohibition of pasture-application in some countries, which could reduce the potential contamination of animal products.

Other management practices such as depth of mixing into the soil and losses through various environmental processes will also affect chemical concentrations in soils after land application. Degradation is an important component of loss, but may be incomplete or slow, even for relatively easily degraded chemicals such as linear alkyl benzene sulfonates (LAS). LAS is present at such high concentrations in sludges (up to $3 \%$ by weight) that incomplete degradation coupled with repeated applications could result in consistently elevated LAS concentrations in soils. This was demonstrated in one study that detected over $10 \mathrm{mg} / \mathrm{kg}$ six years after land application of sludge. Importantly, no further decrease was found after two more years, indicating that the residual LAS was resistant to degradation (Carlsen et al., 2002).

\section{Conclusion}

More data are needed on the chemicals that are in sludges today and on the temporal trends for those chemicals. Relying on existing lists of chemicals such as priority pollutants will not identify many chemicals of current concern.

To make more informed assessments about the impact of sludge processing on chemical concentrations, more information on the type of treatment (both of the wastewater and the sludge) and the sludge residence time as well as the nature of significant nondomestic inputs is needed. Detection methods and limits of detection need to be reported. Where multiple samples are analyzed, individual data points as well as median and means should be reported since averaging values among several sludges may obscure information relating to the differences due to inputs or treatment.

This paper demonstrates that there are groups of chemicals for which there are relatively abundant sludge concentration data (such as PCBs, pesticides and PAHs), while there are others for which few data have been collected (such as nitrosamines). Certain classes of chemicals also are shown to have high percentage of reported concentrations that exceed SSLs, suggesting that analysis of additional chemicals in those classes may be warranted. Few data exist on the fate of sludgeborne chemicals in field soils and such research is critical to assessing the risks posed by sludge application.
Evaluating the risks posed by individual chemicals, let alone mixtures requires multiple assumptions that can lead to unacceptably high levels of uncertainty. Current limitations in our knowledge base regarding the amount and type of chemicals in sludges exacerbate this problem, as does the limited availability of fate and toxicity data, for both human and non-human receptors. As sludge application occurs on farms, forests, and mines, as well as residential and recreational land, humans, wildlife and soil organisms may all be exposed to the organic contaminants present in sludges. Filling the gaps in knowledge regarding the concentration, fate and toxicity of sludge-borne contaminants is critical if the risks associated with land application are to be adequately characterized.

\section{Appendix A. Supplementary data}

Supplementary data associated with this article can be found, in the online version, at doi:10.1016/j. scitotenv.2006.04.002.

\section{References}

Beck AJ, Alcock RE, Wilson SC, Wang M-J, Wild SR, Sewart AP, et al. Long-term persistence of organic chemicals in sewage sludge-amended agricultural land: a soil quality perspective. Adv Agron 1995;55:345-91.

Bodzek D, Janoszka B. Comparison of polycyclic aromatic compounds and heavy metals contents in sewage sludges from industrialized and non-industrialized region. Water Air Soil Pollut 1999;111:359-69

Bolz U, Hagenmaier H, Korner W. Phenolic xenoestrogens in surface water, sediments, and sewage sludge from Baden-Wurttemberg, south-west Germany. Environ Pollut A 2001;115:291-301.

Carlsen L, Metzon M-B, Kjelsmark J. Linear alkylbenzene sulfonates (LAS) in the terrestrial environment. Sci Total Environ 2002;290: 225-30.

Chaney RL, Ryan JA, O'Connor GA. Organic contaminants in municipal biosolids: risk assessment, quantitative pathways analysis, and current research priorities. Sci Total Environ 1996;185(1-3):187-216.

Constable TW, Taylor LJ, Rush RJ. The effect of three sludge processing operations on the fate and leachability of trace organics in municipal sludges. Environ Technol Lett 1986;7:129-40.

Environment Canada. Assessment report-nonylphenol and its ethoxylates. Environment Canada; 2004.

European Commission Joint Research Centre. Technical guidance document on risk assessment. Part II. EUR 201418 EN/2. Institute for Health and Consumer Protection; 2003.

Fries GF. Ingestion of sludge applied organic chemicals by animals. Sci Total Environ 1996;185(1-3):93-108.

Jobst H. Chlorophenols and nonylphenols in sewage sludges. Part II: did contents of pentachlorophenol and nonylphenols reduce? Acta Hydrochim Hydrobiol 1998;26(6):344-8.

Keith LH, Telliard WA. Pollutants I-a perspective view. Environ Sci Technol 1979;13:416-23. 
Ministry of Environment and Energy. Statutory order from the ministry environment and energy no. 823 of September 16, 1996, on application of waste products for agricultural purposes. Danish Environmental Protection Agency; 1997.

National Research Council. Use of reclaimed water and sludge in food crop production. Washington, DC: National Academy Press; 1996. 178 pp.

National Research Council. Biosolids applied to land. Washington, DC: National Academy Press; 2002.

Netherlands Ministry of Housing Spatial Planning and Environment. Circular on target values and intervention values for soil remediation; 2000. p. 51. http://www2.minvrom.nl/Docs/internationaal/ annexS_I2000.pdf.

New York State Department of Environmental Conservation. 6 NYCRR Part 360-5 solid waste management facilities. Composting and other class a organic waste processing facilities; 2003.

Ontario Ministry of the Environment. Soil, ground water and sediment standards for use under Part XV.1 of the Environmental Protection Act. Canada: Queen's Printer for Ontario; 2004. p. 39.

Petrasek AC, Kugelman IJ, Austern BM, Pressley TA, Winslow LA, Wise RH. Fate of toxic organic compounds in wastewater treatment plants. J Water Pollut Con Fed 1983;55(10):1286-96.

Pryor SW, Hay AG, Walker LP. Nonylphenol in anaerobically digested sewage sludge from New York State. Environ Sci Technol 2002;36 (17):3678-82.

Rogers HR. Sources, behaviour and fate of organic contaminants during sewage treatment and in sewage sludges. Sci Total Environ 1996;185:3-26.

Schnaak W, Kuchler T, Kujawa M, Henschel K-P, Subenbach D, Donau R. Organic contaminants in sewage sludge and their ecotoxicological significance in the agricultural utilization of sewage sludge. Chemosphere 1997;35(1-2):5-11.

Schowanek D, Carr R, David H, Douben P, Hall J, Kirchmann H, et al. A Risk-based methodology for deriving quality standards for organic contaminants in sewage sludge of use in agricultureconceptual framework. Regul Toxicol Pharmacol 2004;40(3): 227-51.

Scrimshaw MD, Langford KH, Lester JN. Analytical methods for the determination of alkylphenolic sufactants and polybrominated duphenyl ethers in wastewaters and sewage sludges. I. A review of methodologies. Environ Technol 2004;25(8):967-74.

Ternes TA, Joss A, Siegrist H. Scrutinizing pharmaceuticals and personal care products in wastewater treatment. Environ Sci Technol 2004;38(20):393A-9A.
U.S. Environmental Protection Agency. National sewage sludge survey; availability of information and data, and anticipated impacts on proposed regulations; proposed rule. Part III. Fed Regist 1990;55(218):47210-83.

U.S. Environmental Protection Agency. A guide to the biosolids risk assessments for the EPA Part 503 rule. EPA832-B-93-005. U.S. EPA, Office of Wastewater Management; 1995. p. 144.

U.S. Environmental Protection Agency. Technical support document for the round two sewage sludge pollutants. EPA-822-R-96-003. Washington: Office of Water, Office of Science and Technology, Health and Ecological Criteria Division; 1996.

U.S. Environmental Protection Agency. Biosolids generation, use and disposal in the United States. Municipal and Industrial Solid Waste Division, Office of Solid Waste; 1999. EPA530-R-99-009, $74 \mathrm{pp}$.

U.S. Environmental Protection Agency. Exposure analysis for dioxins, dibenzofurans, and coplanar polychlorinated biphenyls in sewage sludge. Washington: Office of Water; 2002.

U.S. Environmental Protection Agency. Standards for the use or disposal of sewage sludge: decision not to regulate dioxins in landapplied sewage sludge. Fed Regist 2003a;68(206):61083-96.

U.S. Environmental Protection Agency. Standards for the use or disposal of sewage sludge; final agency response to the national research council report on biosolids applied to land and the results of EPA's review of existing sewage sludge regulations. Fed Regist 2003b;68(250):75531-52.

U.S. Environmental Protection Agency. Superfund analytical services/Contract Laboratory Program (CLP). http://www.epa. gov/superfund/programs/clp/target.htm; 2004.

U.S. Environmental Protection Agency Superfund. Soil screening guidance. http://www.epa.gov/superfund/resources/soil/appd_a. pdf;1996 EPA/540/R-95/128.

Wild SR, Jones FW. The effect of sludge treatment on the organic contaminant content of sewage sludges. Chemosphere 1989;19 (10-11):1765-77.

Wild SR, Jones KC. Organic chemicals entering agricultural soils in sewage sludges: screening for their potential to transfer to crop plants and livestock. Sci Total Environ 1992;119:85-119.

Wild SR, Berrow ML, McGrath SP, Jones KC. Polynuclear aromatic hydrocarbons in crops from long-term field experiments amended with sewage sludge. Environ Pollut 1992;76:25-32.

Zitomer DH, Speece RE. Sequential environments for enhanced biotransformation of aqueous contaminants. Environ Sci Technol 1993;27(2):226-43. 